\title{
Simulation of Cooperative Automated Driving by Bidirectional Coupling of Vehicle and Network Simulators
}

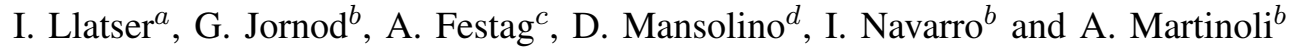

\begin{abstract}
The convergence of sensor-based vehicle automation and Inter-Vehicle Communication (IVC) will be a key to achieve the full automation of vehicles. In this paper we present a new method for the design and performance evaluation of Cooperative Automated Driving (CAD) systems, based on a bidirectional coupling of vehicle and network simulators (Webots and ns-3). The coupling exploits the comprehensive capabilities of the simulators at a reasonable computational complexity and allows simulating CAD systems with high accuracy. We demonstrate the capabilities of the simulation tool by a case study of convoy driving with automated vehicles using a fully distributed control algorithm and IVC. The study compares CAD-specific metrics (safety distance, headway, speed) for an ideal and a realistic communication channel. The simulation results underline the need of accurate modeling and give valuable insights for the design of CAD systems.
\end{abstract}

\section{INTRODUCTION}

Today's commercial vehicles are equipped with various Advanced Driver Assistant Systems (ADAS) that improve driving safety, traffic efficiency and comfort. These systems rely on vehicle sensors, such as radar or cameras, which continuously observe the vehicle's environment in real-time. Combined with the vehicle control, ADAS realize already today a certain level of automated driving, where the vehicle driver is relieved from selected control tasks. A typical example is the combination of power steering with adaptive cruise control and lane keeping, which allows for automated highway driving with lateral and longitudinal vehicle control.

IVC enables nearby vehicles to behave cooperatively by exchanging information with each other. This allows overcoming limitations of the vehicle sensors, such as their restricted field of view, perception range and susceptibility to adverse weather conditions. IEEE 802.11p-based IVC technology operating in the $5.9 \mathrm{GHz}$ frequency band, targeting use cases for driver information and warning, is currently standardized, mature and ready to be deployed [1].

This work was supported by the European Commission under AutoNet2030, a collaborative project part of the Seventh Framework Programme for research, technological development and demonstration (Grant Agreement NO. 610542). The authors would like to thank all partners in the AutoNet2030 project for their cooperation and valuable contribution.

${ }^{a}$ I. Llatser is with Robert Bosch GmbH, Hildesheim, Germany. Email: ignacio.llatserede.bosch.com

${ }^{b}$ G. Jornod, I. Navarro and A. Martinoli are with the Distributed Intelligent Systems and Algorithms Laboratory (DISAL), School of Architecture, Civil and Environmental Engineering, École Polytechnique Fédérale de Lausanne (EPFL), Lausanne, Switzerland.

${ }^{c}$ A. Festag is with Fraunhofer Institute for Transportation and Infrastructure Systems IVI, Dresden, Germany.

${ }^{d}$ D. Mansolino is currently with Cyberbotics Ltd. although he performed this work entirely at DISAL, his former affiliation.
In the near future, IVC will gradually support higher vehicle cooperation and automation. A next step is the exchange of sensor data among vehicles, which will enable cooperative perception in order to enhance the vehicles' environmental model. Further, the maneuver coordination among vehicles will allow them to synchronize their driving patterns, thus improving their safety. In the long term, it is foreseen that CAD will enable fully autonomous driving, which no longer requires the supervision by human drivers [2], [3].

For the design of CAD, simulations are indispensable and commonly accepted in the research community. With this purpose, a number of highly customized vehicle and network simulation tools with varying abstraction levels and modeling accuracy are currently available. However, being focused in either vehicle or network simulation, existing tools do not provide the level of realism required for the evaluation of advanced CAD systems in their integrity. In this paper, we propose a bidirectionally coupled vehicle and network simulator, which allows recreating CAD scenarios with both highly accurate vehicle dynamics and a realistic communications model. The coupling facilitates to reuse the comprehensive capabilities of existing simulators at a reasonable computational complexity for all CAD levels. In order to demonstrate the potential of our approach, we apply the coupled simulators to the advanced and challenging use case of convoy driving with automated vehicles using a fully distributed control algorithm and IVC.

The contributions of the present paper are three-fold. First, we review the state-of-the-art of bidirectionally coupled simulators for IVC. Second, we propose a new simulation framework for IVC that bidirectionally couples advanced vehicle and network simulators to accurately evaluate CAD systems. Third, we evaluate the maneuvering performance of a vehicle convoy with the proposed framework as a case example. The product of this work is an open-source plug-in ${ }^{1}$ enabling the use of ns-3 [4] within Webots [5]. The present work relies on and extends our work on distributed control of convoy driving [6] and on the performance evaluation of the communication system in convoys [7]. Compared to our previous work, this paper addresses convoy maneuvering supported by IVC and investigates the impact of IVC parameters on the maneuvering performance of a graph-based control algorithm for automated convoy driving. The decentralized control scheme of convoys allows a better scalability with respect to experimentally demonstrated vehicle platoons [8].

\footnotetext{
${ }^{1}$ The plug-in is available at https://github.com/epfl-disal/ ns34webots.
} 
The remainder of this paper is organized as follows. Sec. II gives an overview of existing simulation frameworks for IVC, followed by details of the proposed vehicle and network simulator in Sec. III. Sec. IV presents the case study for vehicle convoys that applies the proposed simulator, including a description of the convoy driving algorithm, the simulation scenario and parameters, and the discussion of the simulation results. Finally, Sec. V draws conclusions.

\section{Simulation FRAMEWORKS FOR IVC}

A simulation tool specifically designed for CAD systems does not exist to date. On the one hand, network simulators provide an accurate model of the communication aspects, but they offer simple mobility patterns for the simulation nodes (such as the random waypoint model) which do not reflect the complex maneuvers of vehicles, especially in urban scenarios. On the other hand, vehicle and road traffic simulators allow a realistic reproduction of the vehicle dynamics and real-life traffic conditions; however, they typically include simplistic models (if any) for IVC.

Extending either of these simulators to include good models for both mobility and IVC would be very complex. Instead, a popular alternative is to take a modular approach by combining a network and a microscopic traffic simulator to profit from their respective strengths. A first approach is an offline combination, for instance, by generating vehicle traces with a traffic simulator and using them as input for the network simulator.

A more advanced option is the bidirectional coupling of simulators, where several simulators run in parallel and exchange data among them in real time. The most prominent of such approaches are described as follows.

Veins [9] is an open source framework based on the simulators OMNeT++ and SUMO. OMNeT++ is a network simulator which includes a wide range of models for IVC, such as IEEE 802.11p and IEEE 1609.4 DSRC/WAVE, while SUMO is a widely used microscopic traffic simulator which implements realistic vehicle-following models and allows importing real-life map data.

iTETRIS [10] is an open source modular architecture that integrates a traffic and a network simulator, with SUMO and ns-3 as a case example. A complete model of the IVC networking stack (according to the ETSI standards) and the wireless channel are implemented. iTETRIS supports the implementation of IVC-based applications in a languageagnostic fashion.

TraNS [11] was one of the main attempts to integrate traffic and network simulators (SUMO and ns-2) in a GUI tool. Released in 2007, it is currently discontinued.

VSimRTI [12] follows a flexible simulator coupling concept which incorporates different network simulators, such as ns-3, OMNeT++, JiST/SWANS and the VSimRTI cellular network simulator, with the traffic simulators SUMO and PHABMACS. Further simulators can also be integrated by implementing the corresponding interface. Similarly to iTETRIS, the focus is on the evaluation of IVC applications over a network architecture based on the ETSI ITS standard.
Even though these coupled simulators provide a good framework to test IVC applications, the vehicle movements are in all cases provided by microscopic traffic simulators, which are best suited to model large-scale scenarios. Indeed, a microscopic traffic simulator is capable of simulating hundreds of vehicles driving in a whole city in real time, but it does not include a precise model of the individual vehicle dynamics (such as the transmission system or the tire grip). Therefore, we propose next the novel approach of combining a submicroscopic vehicle and a network simulator, in order to achieve the highest possible degree of realism in small-scale CAD scenarios.

\section{COUPLED VEHICLE AND NETWORK SIMULATOR}

Our new simulation framework combines the simultaneous execution of a submicroscopic vehicle simulator implemented in Webots and the network simulator ns-3, integrated as a communication plug-in for the vehicle simulator.

Webots is a powerful, submicroscopic, high-fidelity simulator based on the Open Dynamics Engine (ODE) library. It was originally developed for mobile robotics and later extended to support simulation of intelligent vehicles [13], [14]. It provides several models of vehicles, which have been fully calibrated and validated by running benchmarks both in simulation and using real vehicles and comparing their dynamics. Furthermore, Webots contains various sensor models typically used in the automotive research and industry, such as cameras, lidar and GPS. A previous effort bridging OMNeT++ with Webots has been presented in [15].

ns-3 is a discrete-event network simulator widely used for the modeling and evaluation of communication networks and Internet systems. ns-3 is particularly well suited for the simulation of IVC due to its highly accurate model of the WiFi network stack and the IEEE 802.11 p physical $(5.9 \mathrm{GHz}$ frequency band) and MAC layers.

Both simulators are combined by means of a plug-in channel which allows the information exchange between the simulators in real time. The following steps constitute the workflow of our proposed framework:

1) At the simulation start, Webots initializes the vehicle nodes and sets their initial positions, headings and speeds. Each vehicle is assigned a controller which determines its lateral and longitudinal control commands along the simulation. At the same time, the plug-in creates and initializes the corresponding network nodes in ns-3 with the respective positions.

2) At every simulation step, Webots updates the positions of all vehicles according to the output of the controller and dynamics of the vehicle. The communication plug-in updates the position of the ns-3 nodes accordingly and notifies the network simulator about any transmitted messages.

3) If any vehicle receives a message during the simulation step, the network simulator uses a callback function in the plug-in to notify the vehicle controller. The controller uses the received information to compute the control commands.

By repeating the last two steps, we are able to perform bidirectionally coupled simulations with both vehicle and 
TABLE I

SIMULATION TIME INCREASE [\%] WHEN USING THE NS-3 PLUG-IN

\begin{tabular}{|c|c|c|c|c|c|c|}
\hline \multirow[b]{2}{*}{ \# vehicles } & \multicolumn{6}{|c|}{ Message frequency } \\
\hline & $15 \mathrm{~Hz}$ & $10 \mathrm{~Hz}$ & $5 \mathrm{~Hz}$ & $2 \mathrm{~Hz}$ & $1 \mathrm{~Hz}$ & $.5 \mathrm{~Hz}$ \\
\hline 2 & 12.25 & 23.27 & 23.03 & 14.43 & 7.84 & 1.47 \\
\hline 3 & 78.03 & 56.59 & 20.07 & 20.64 & 28.45 & 5.1 \\
\hline 6 & 45.93 & 14.73 & 3.83 & 14.09 & 71.8 & 65.83 \\
\hline 12 & 278.96 & 8.85 & 61.01 & 96.51 & 19.86 & 62.49 \\
\hline 18 & 406.1 & 274.59 & 34.68 & 154.21 & 173.01 & 167.75 \\
\hline 24 & 316.45 & 199.96 & 179.17 & 91.9 & 170.19 & 121.07 \\
\hline
\end{tabular}

TABLE II

SELECTED EXECUTION TIMES [S] WITHOUT / WITH THE PLUG-IN AND THE CORRESPONDING SPEED-UP FACTORS [X]

\begin{tabular}{|c|c|c|c|c|}
\hline \multirow[b]{2}{*}{ \# vehicles } & \multicolumn{4}{|c|}{ Message frequency } \\
\hline & $15 \mathrm{~Hz}$ & $.5 \mathrm{~Hz}$ & $15 \mathrm{~Hz}$ & $.5 \mathrm{~Hz}$ \\
\hline 2 nodes & $2.43 / 2.73$ & $2.63 / 2.66$ & x16.46/x14.67 & $\mathrm{x} 15.24 / \mathrm{x} 15.02$ \\
\hline 24 nodes & $35.12 / 146.27$ & $15.93 / 35.23$ & $\mathrm{x} 1.14 / \mathrm{x} 0.27$ & $\mathrm{x} 2.51 / \mathrm{x} 1.14$ \\
\hline
\end{tabular}

network simulators. As opposed to previous combined simulation efforts, our proposed approach allows the use of very realistic models for both the vehicle control and the communication protocols. Subsequently, the obtained results benefit from a high-fidelity multi-vehicle simulator combined with a realistic network simulator.

This combination enables designers of networked robots or automated vehicles to account for realistic communication in their conceptions with small computational overhead. Table I shows the time percentage increase when using the presented ns-3 plug-in with respect to an ideal model of the communication by default implemented in Webots. This computational overhead is measured as the percentage increase in simulation time in fast mode (without 3D graphics rendering) over 50 simulation occurrences with different values of message frequency and number of nodes. Table II provides a reference for the simulation times in fast mode of the $40 \mathrm{~s}$ experiment on a desktop computer with an Intel Core i7 CPU (8 cores @ $3.5 \mathrm{GHz}$ ) running one instance of Webots at the time in Ubuntu 14.04.

\section{Case Study: Convoys of Automated Vehicles}

In this section, we present an application of the proposed vehicle and network simulator.

\section{A. Convoy Control and Communication Algorithms}

In this paper, convoys are groups of automated vehicles which maintain a formation ruled by a distributed graphbased algorithm and information exchange via IVC [6]. As opposed to ACC with reactive spacing control, convoys in this scope are leaderless formations in which vehicles cooperatively adapt their inter-vehicle distance to maintain the desired configuration and common group speed.

The distributed graph-based algorithm is based on Laplacian control [16]. Each vehicle maintains a graph whose vertices represent vehicles and whose edges consist of the communication links [6], solely using the broadcast relative position of its neighbors. Using this graph and the Laplacian

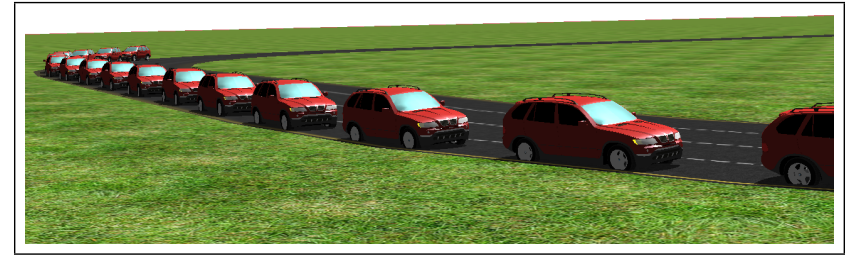

Fig. 1. A single-lane convoy of automated vehicles in Webots

control, each agent computes its target speed to maintain the desired configuration. We use the lateral controller defined by [17], intended to remove angular and lateral errors and ensure lane keeping. Our previous works [6] fully describes the distributed graph-based control, usage of curvilinear coordinates, longitudinal and lateral controllers, as well as other implementation details.

In order to maintain the local graphs and to perform lanechange maneuvers in the case of multi-lane convoys, convoy controllers need to exchange kinematic state information such as position, velocity and heading, as well as maneuver intentions. This exchange is achieved through single-hop broadcast of custom convoy messages using IVC. Given the typical transmission range of WiFi-based IVC, the neighbor vehicles in a convoy are located within the single-hop range, unless this range is artificially restricted to reduce the graph computations.

Three types of convoy messages have been defined. First, convoy vehicles transmit periodically info messages to exchange kinetic state information with their neighbors. The transmission frequency of info messages is a design parameter, which depends on the convoy size, speed and the specific vehicle formation, and it will have a direct impact on the performance of the formation control algorithm. In addition, the brake message is transmitted by a convoy vehicle whenever it requires to apply the brakes (e.g., due to traffic congestion ahead). The endbrake message is correspondingly broadcast after a brake message to indicate that a vehicle is not braking anymore. These messages enable cooperative maneuvering among convoy vehicles and allow them to improve their reaction to sudden brake maneuvers of preceding vehicles and therefore prevent the appearance of string instabilities.

Convoy vehicles are designed to brake when receiving a brake message from a vehicle ahead, but ignore the messages from vehicles behind. Since vehicles only account for front sourced brake messages and their propagation is ended by an endbrake message reception, any oscillation phenomena due to back-propagation of the message are avoided.

\section{B. Simulation Scenario}

We consider a scenario of a single-lane convoy with a given number of vehicles (which varies from 2 to 24 ). The leading vehicle brakes periodically for $2 \mathrm{~s}$ every $10 \mathrm{~s}$, simulating a congested traffic scenario. This allows evaluating how the following convoy vehicles adapt to the speed changes of the leading vehicle.

The vehicles are controlled by the convoy algorithm described in Sec. IV-A, implemented in Webots (v8.4.0), which allows them to perform lane-keeping and cooper- 
TABLE III

SIMULATION PARAMETERS

\begin{tabular}{l|l} 
Parameter & Values \\
\hline \hline Freeway length / shape & $1 \mathrm{~km} /$ Oval \\
Convoy size & $2,3,6,12,18,24$ vehicles \\
Convoy message size & 2,048 bytes \\
Neighbor range & $100 \mathrm{~m}$ \\
Speeds-min, target, max & $13.88,16.66,19.44 \mathrm{~m} / \mathrm{s}$ \\
Convoy message frequency & $0.5-15 \mathrm{~Hz}$ \\
PHY / MAC protocol & ITS-G5 (IEEE $802.11 \mathrm{p})$ \\
Data rate & $6 \mathrm{Mbit} / \mathrm{s}$ \\
Transmit power + antenna gain & $20 \mathrm{dBm}+9.1 \mathrm{~dB}$ \\
Receiver sensitivity & $-95 \mathrm{dBm}$ \\
Channel bandwidth & $10 \mathrm{MHz}$ at $5.9 \mathrm{GHz}$ \\
Experiments replication & $50 \mathrm{times}$ \\
Simulation duration / timestep & $40 \mathrm{~s} / 64 \mathrm{~ms}$
\end{tabular}

ative maneuvering in a fully distributed manner through the exchange of convoy messages. Convoy messages are transmitted as single-hop broadcast messages with the Basic Transport Protocol (BTP) over GeoNetworking. The physical and MAC layers follow the ITS-G5 (European version of IEEE 802.11p) standard, with a single channel and standard parameters for IVC. The radio propagation model considered is a log-distance path loss model with Nakagami fading, with experimentally validated parameters [18].

The convoy communication protocol is modeled in the network simulator ns-3, version 3.25. A simulation run lasts $40 \mathrm{~s}$, the results are calculated as the average of 50 simulation runs (the first $10 \mathrm{~s}$ are not considered in order to allow reaching a steady state regime). The time instant of the first transmission is randomly chosen by each vehicle in the interval $[0, T]$, where $T$ is the message period in ms, to cope with the fact that Webots simulation works simultaneously while ns-3 is a discrete-event simulator. The main simulation parameters are summarized in Table III.

We study the convoy maneuvering performance as a function of three simulation parameters: ( $i$ ) the number of vehicles in the convoy, (ii) the transmission frequency of convoy info messages, and (iii) the inter-vehicle safety distance of the convoy controller. By varying these parameters, we can find out which combination results in the best performance and which situations challenges the most the convoy control algorithm. To provide a comparison baseline, each experiment is first performed with a perfect communication setup, in which the communication among vehicles happens in ideal conditions, i.e., no packet loss and no other delay than the simulation timestep, $64 \mathrm{~ms}$.

\section{Performance Metrics}

The considered metrics to evaluate the performance of the convoy maneuvering are the following:

The safety distance ratio is defined as the percentage of convoy vehicles whose inter-vehicle distance is over a specified threshold. A low safety distance ratio indicates that the convoy vehicles are not able to keep the minimum safety distance with respect to the vehicle in front (e.g., due to outdated data about their neighbor vehicles).

The inter-vehicle distance is defined as the distance from the back bumper of one convoy vehicle to the front bumper of the following vehicle. The inter-vehicle distance is calculated for every convoy vehicle (except the last one, which obviously has no vehicle behind). The inter-vehicle distance of every convoy vehicle should be as close as possible to the targeted safety distance.

The vehicle speed; an average speed as close as possible to the desired speed indicates a higher performance of the convoy since it reflects a fast reaction to the perturbation.

In summary, the best performance is achieved with the combination of a large safety distance ratio, an inter-vehicle distance and a vehicle speed as close as possible to the set targets. Let us explore next how these metrics depend on the system design parameters mentioned above (communication channel model, message frequency, number of vehicles in the convoy and inter-vehicle safety distance).

\section{Simulation Results}

In what follows, we consider first an ideal communication model (all transmitted messages are instantaneously received by all vehicles without errors), and later show the influence of a realistic communication model using ns-3. To avoid repetition, we present and discuss the results for an intervehicle safety distance of 10 meters, as they better represent the trends observable for other values of this parameter. The plots show the averaged results with $95 \%$ confidence intervals.

Our analysis guidelines for both communication models is the following: first, the safety distance ratio is observed and parameter sets showing a safety distance ratio under $80 \%$ are discarded; second, the other metrics are then analyzed for the remaining parameters; third, overlapping sets between communication models are compared.

1) Perfect communication model: Fig. 2a shows the average safety distance ratio of convoy vehicles for the perfect communication model. We observe that message frequencies over $2 \mathrm{~Hz}$ result in no safety distance breaches. An exception is the 6-vehicles convoy at $5 \mathrm{~Hz}$, due to a string instability [19] which starts at the initialization and is then amplified by the brakes (this phenomena is observed in the simulator along the runs). At low message frequencies, small convoys are the most affected as they are constrained by fewer vehicles. For instance, at $0.5 \mathrm{~Hz}$ in a two-vehicle convoy, the behavior of the following vehicle is only influenced by the front vehicle, from which it receives no messages for almost two seconds. This results in numerous safety distance breaches. This argument also explains why larger convoys are less affected by the decrease in message frequency in terms of safety distance ratio, which stays over $90 \%$. Following our analysis guidelines, we discard small convoys with message frequency under $2 \mathrm{~Hz}$ for the remaining of this section.

In the results illustrated by Fig. $2 b$, the inter-vehicle distance exceeds the target safety distance of 10 meters in most of the studied cases. Most values are in the range 9 to 11.5 meters, which is an acceptable interval. Larger convoys 


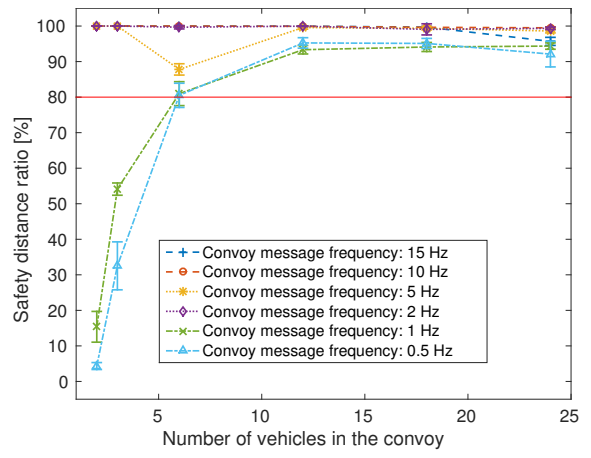

(a)

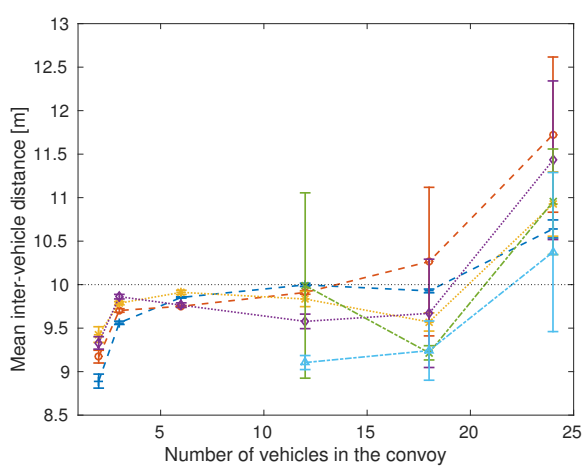

(b)

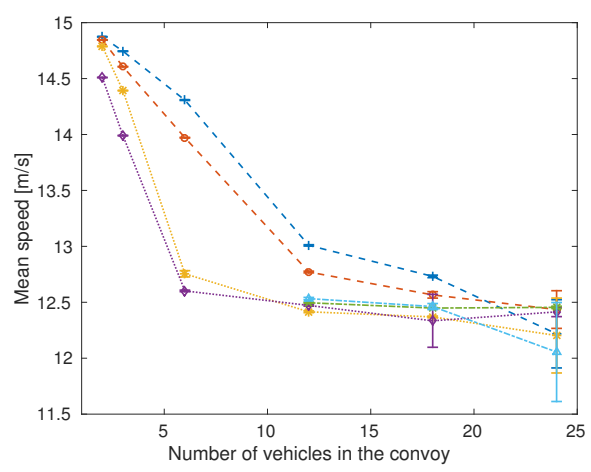

(c)

Fig. 2. Perfect communication model: Comparison of (a) the average safety distance ratio, (b) the average inter-vehicle distance, (c) the average speed in a convoy as a function of the number of vehicles in the convoy, for different values of the convoy message frequency. Note that following our analysis guidelines, parameter sets showing a safety distance ratio under $80 \%$ (i.e. 6 vehicles and less from plot (a)) are discarded in plots (b) and (c).

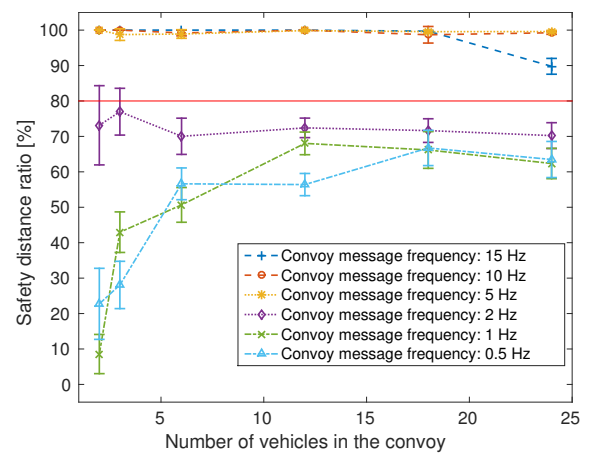

(a)

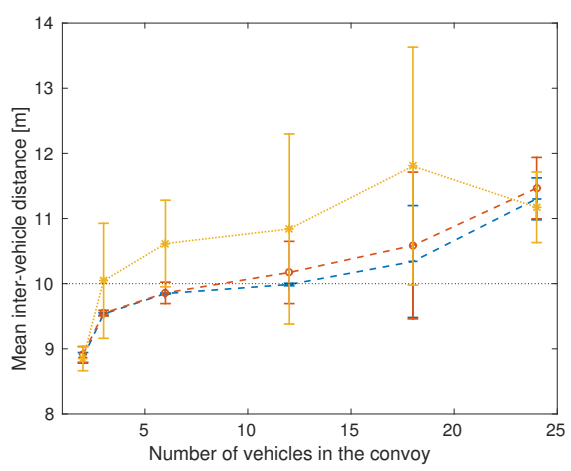

(b)

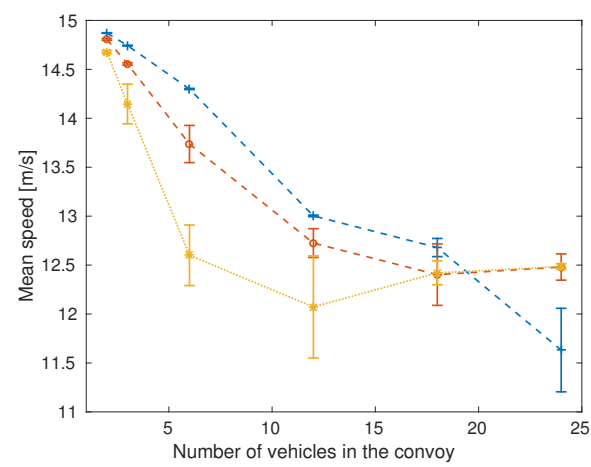

(c)

Fig. 3. Realistic communication model: Comparison of (a) the average safety distance ratio, (b) the average inter-vehicle distance, (c) the average speed in a convoy as a function of the number of vehicles in the convoy, for different values of the convoy message frequency. Note that following our analysis guidelines, parameter sets showing a safety distance ratio under $80 \%$ (i.e. convoy frequency below $5 \mathrm{~Hz}$ in plot (a)) are discarded in plots (b) and (c).

result in higher deviations from the target value and between runs, due to the larger number of vehicles influencing the mean as well as the loose nature of the convoy (which adapts to the road geometry, here an oval). Decreasing the message frequency does not have a clear influence on the average inter-vehicle distance. Together with the large error bars, this suggests that the metric difference for the same convoy size is due to the randomization at the initialization and not to the decrease of message frequency.

Fig. 2c shows the mean speed of all convoy vehicles over last $30 \mathrm{~s}$ of the experiment. We observe that no convoy achieved the target speed of $16.66 \mathrm{~m} / \mathrm{s}(60 \mathrm{~km} / \mathrm{h})$. This is expected considering that $(i)$ the speed is controlled in an open-loop [6]; (ii) the convoy periodically brakes to simulate traffic congestion conditions; and (iii) in our communication approach, messages are broadcast without feedback. There is a clear inversely proportional trend between the number of vehicles in the convoy and the mean speed, which reaches $12 \mathrm{~m} / \mathrm{s}$ for very large convoys. The convoy speed also shows a clear dependence on the message frequency. Indeed, for all data points except for the largest convoys, the larger the message frequency, the slower the convoy. This observation suggests that the outdated information tends to refrain the convoy from reaching higher speeds.
2) Realistic communication model: Fig. 3a presents the safety distance ratio as a function of the number of vehicles in the convoy with different convoy message frequencies using the coupled vehicle and network simulator. For message frequencies strictly over $1 \mathrm{~Hz}$, the convoy size almost does not affect the safety distance ratio. Compared to the perfect case, we observe a safety distance ratio decrease for very large convoys with $15 \mathrm{~Hz}$ message frequency. Furthermore, there is a significant performance drop when decreasing the frequency from 5 to $2 \mathrm{~Hz}$. Finally, for smaller frequencies ( 1 and $0.5 \mathrm{~Hz}$ ), the safety distance ratio increases with the convoy size. This behavior can also be explained by the robustness brought by a larger number of vehicles when a communication link fails. Discarding combinations of parameters showing a distance ratio under $80 \%$, the following analysis focuses on the results with frequencies of 5 to $15 \mathrm{~Hz}$.

The observed mean inter-vehicle distance is close to the target of $10 \mathrm{~m}$, as illustrated by Fig. 3b. The results are consistent with the expected effect of larger convoys. Moreover, decreasing the message frequency from 10 to $5 \mathrm{~Hz}$ increases significantly the average distance between vehicles.

Fig. $3 \mathrm{c}$ represents the average vehicle speed for the realistic communication case. There is a clear decrease of the mean convoy speed as the vehicles number grows. However, this decrease is slower with a larger message frequency. The 
speed values seem to converge for larger convoys to a value of $12.5 \mathrm{~m} / \mathrm{s}$, except for the highest message frequency and convoy size case. The lower mean speed in this scenario is due to the congestion of the communication channel [7] and is consistent with the sudden decrease in the safety distance ratio for the 18 vehicles convoy at $15 \mathrm{~Hz}$ (see Fig. 3a).

3) Model comparison and discussion: Following our analysis guidelines, we compare the values corresponding to a safety distance ratio over $80 \%$. According to this criteria, the experiments with realistic communication indicate that only frequencies under $2 \mathrm{~Hz}$ for small convoys present a safety risk. However, the perfect model shows that, with the implemented control law, a minimum frequency of $5 \mathrm{~Hz}$ is required for convoy formation and maintenance independently of the convoy size; besides, increasing the communication frequency does not improve the performance. Hence, the perfect model provides over-optimistic results in this case.

Moreover, the randomization effect on the mean intervehicle distance is less clear in the realistic model, due to the smaller number of data points, but is still observable. Finally, the slower decrease of mean speed as a function of increasing number of vehicles for higher message frequencies still holds the realistic model, with the exception of the highest frequency with the largest convoy.

In a general manner, the trends observed in the perfect communication model also hold for the realistic one, although the latter shows a less optimistic performance-with maximum absolute differences on average of 38.85 percentage points, $23.34 \%$ and $4.8 \%$ in terms of safety distance ratio, vehicle speed and inter-vehicle distance, respectively. Indeed, the existence of a threshold in terms of message frequency that affects smaller convoys, the slower drop in mean speed with high frequency communication and the rather small effect of message frequency on the average intervehicle distance are observed in both models. However, the packet losses and latency taken into account by the realistic model require the vehicle control algorithm to work with more outdated information about its neighbors, which leads to a drastically higher number of safety distance breaches (compare Fig. 2a with Fig. 3a).

\section{Conclusions}

In this paper, we have proposed a novel tool to design and analyze CAD systems, namely, a bidirectionally coupled vehicle and network simulation framework. We have implemented an open-source communication plug-in to connect the vehicle simulator Webots with the network simulator ns-3. As opposed to existing approaches considering combinations of traffic and network simulators, our proposed simulator allows the analysis of complex CAD scenarios with higher realism.

In order to prove its usefulness for designers of CAD systems, we have demonstrated the capabilities of the coupled simulator to evaluate the maneuvering performance of a convoy of automated vehicles. In particular, the maintenance of the safety distance, the average headway and the average speed of convoy vehicles are measured as a function of the convoy message frequency and the number of vehicles. The results obtained with a perfect communication model are used as a baseline to evaluate the impact of realistic IVC in the convoy performance. In general, we find that the perfect communication model over-estimates the performance of the convoy algorithm-especially in terms of safety distance ratio-with respect to the realistic model (see Sec. IVD.3). Moreover, the computational overhead of the realistic model is negligible for small-scale convoys, whereas it scales reasonably well in larger scenarios.

\section{REFERENCES}

[1] A. Festag, "Cooperative Intelligent Transport Systems Standards in Europe,” IEEE Commun. Mag., vol. 52, no. 12, pp. 166-172, 2014.

[2] L. Hobert et al., "Enhancements of V2X Communication in Support of Cooperative Autonomous Driving," IEEE Commun. Mag., vol. 53, no. 12, pp. 64-70, 2015.

[3] M. Obst et al., "Challenges for Automated Cooperative Driving: The AutoNet2030 Approach," in Automated Driving: Safer and More Efficient Future Driving, 1st ed., D. Watzenig and M. Horn, Eds. Cham: Springer Int. Publishing, 2017, ch. 26, pp. 561-570.

[4] T. Henderson, M. Lacage, and G. Riley, "Network Simulations with the ns-3 Simulator," in ACM SIGCOMM, Seattle, USA, 2008, p. 527.

[5] O. Michel, "Webots: Professional Mobile Robot Simulation," Journal of Advanced Robotics Systems, vol. 1, no. 1, pp. 39-42, 2004.

[6] I. Navarro, F. Zimmermann, M. Vasic, and A. Martinoli, "Distributed Graph-Based Control of Convoys of Heterogeneous Vehicles Using Curvilinear Road Coordinates," in IEEE Intelligent Transportation Systems Conference, Rio de Janeiro, Brazil, 2016, pp. 879-886.

[7] I. Llatser, A. Festag, and G. Fettweis, "Vehicular Communication Performance in Convoys of Automated Vehicles," in IEEE Int. Conference on Communications, Kuala Lumpur, Malaysia, 2016, pp. 1-6.

[8] E. Chan, P. Gilhead, P. Jelinek, P. Krejci, and T. Robinson, "Cooperative control of sartre automated platoon vehicles," in 19th ITS World Congress, Vienna, Austria, 2012, pp. 22-26.

[9] C. Sommer, R. German, and F. Dressler, "Bidirectionally Coupled Network and Road Traffic Simulation for Improved IVC Analysis," IEEE Trans. Mobile Comput., vol. 10, no. 1, pp. 3-15, 2011.

[10] M. Rondinone et al., "iTETRIS: A Modular Simulation Platform for the Large Scale Evaluation of Cooperative ITS Applications," Elsevier Simulation Modelling Practice and Theory, vol. 34, pp. 99-125, 2013.

[11] M. Piórkowski et al., "TraNS: Realistic Joint Traffic and Network Simulator for VANETs," ACM SIGMOBILE Mobile Computing and Communications Review, vol. 12, no. 1, pp. 31-33, 2008.

[12] B. Schünemann, "V2X Simulation Runtime Infrastructure VSimRTI: An Assessment Tool to Design Smart Traffic Management Systems," ACM Computer Networks, vol. 55, no. 14, pp. 3189-3198, 2011.

[13] S. Gowal, Y. Zhang, and A. Martinoli, "A Realistic Simulator for the Design and Evaluation of Intelligent Vehicles," in IEEE Int. Conference on Intelligent Transportation Systems, Madeira, Portugal, 2010, pp. 1039-1044.

[14] EPFL DISAL, "A Flexible and Faithful Simulator for Bridging the Gap between Mobile Robots and Intelligent Vehicles," http://disal.epfl.ch/ research/RO2IVSim, 2013-2016, accessed: 2016-11-18.

[15] C. M. Cianci, J. Pugh, and A. Martinoli, "Exploration of an Incremental Suite of Microscopic Models for Acoustic Event Monitoring Using a Robotic Sensor Network," in IEEE Int. Conference on Robotics and Automation, Pasadena, USA, 2008, pp. 3290-3295.

[16] M. Mesbahi and M. Egerstedt, Graph Theoretic Methods in Multiagent Networks. Princeton University Press, 2010.

[17] M. Linderoth, K. Soltesz, and R. M. Murray, "Nonlinear Lateral Control Strategy for Nonholonomic Vehicles," in American Control Conference, Seattle, USA, 2008, pp. 3219-3224.

[18] T. Abbas, K. Sjöberg, J. Karedal, and F. Tufvesson, "A Measurement Based Shadow Fading Model for Vehicle-to-Vehicle Network Simulations," International Journal of Antennas and Propagation, 2012. [Online]. Available: http://arxiv.org/abs/1203.3370

[19] R. Olfati-Saber and R. M. Murray, "Distributed Cooperative Control of Multiple Vehicle Formations Using Structural Potential Functions," in IFAC World Congress, vol. 15, no. 1, Barcelona, Spain, 2002, pp. 242-248. 